\title{
Quem arrancou essa planta do meu jardim? Argumentos a favor do uso de legendas interlinguais no ensino de língua estrangeira
}

\author{
Helena Santiago Vigata (VIGATA, Helena S.) \\ Professora leitora na Universidade de Brasília - UnB \\ Mestranda em Linguística Aplicada pela Universidade de Brasília - UnB \\ subtitulando@gmail.com \\ Lúcia Maria de Assunção Barbosa (BARBOSA, Lúcia M. A.) \\ Professora Doutora da Universidade Federal de São Carlos - UFSCar \\ Professora convidada no Programa de Pós-Graduação em Linguística Aplicada da UnB \\ lubarbo@ufscar.br
}

\section{Resumo}

O presente artigo propõe uma reflexão sobre o uso de material audiovisual legendado no ensino/aprendizagem de língua estrangeira (LE) com o objetivo de promover a reflexão sobre o potencial pedagógico das legendas interlinguais com vistas ao desenvolvimento da competência intercultural. Para tanto, faz-se uma revisão dos conceitos de tradução pedagógica e competência intercultural e, em seguida, se apresentam algumas bases metodológicas para trabalhar com legendas interlinguais dentro do paradigma comunicativo. Espera-se, com as discussões e propostas aqui apresentadas, contribuir para a desmistificação da tradução como erva daninha para a aquisição de uma nova língua.

Palavras-chave: competência intercultural, tradução pedagógica, legendas interlinguais.

\begin{abstract}
This paper aims to argue in favour of the use of subtitled audiovisual material in foreign language teaching and learning, and, more specifically, to encourage reflections on the pedagogical potential of interlingual subtitles for developing intercultural competence. After a review of the concepts of pedagogical translation and intercultural competence, some guidelines are presented for the use of interlingual subtitles within a communicative paradigm. As a result, this study seeks to contribute to the demystification of translation as a 'weed' in the acquisition of another language.
\end{abstract}

Keywords: intercultural competence, pedagogical translation, interlingual subtitles. 


\section{Introdução}

Decorridos alguns anos desde que se iniciou um movimento em defesa da volta da tradução, como recurso didático, na área de ensino e aprendizagem de línguas desde que seu uso fosse pontual, justificado e condizente com o paradigma comunicativo -, ainda há dificuldades para encontrar propostas metodológicas que ajudem a implementá-la na sala de aula.

A crescente publicação de estudos (RIDD, 2003; HURTADO, 1999; LAVAULT, 1998; COSTA, 1988; FERREIRA, 1999; POPOVIC, 1999; ZABALBEASCOA, 1990) sobre a tradução pedagógica mostra que esta modalidade vem conquistando espaço e respeitabilidade, tanto como procedimento quanto como objeto de pesquisa, mas permaneceram algumas ressalvas que, a nosso ver, restringiram o potencial pedagógico da tradução. Uma delas é a concepção de que as legendas interlinguais prejudicam a aquisição ao interferir de maneira negativa nos níveis de atenção do aprendiz. Esta crença, que não leva em conta os outros aspectos envolvidos do processo, tem provocado uma certa interdição desta modalidade de tradução nas salas de aula. O objetivo deste trabalho é ampliar essa visão reducionista e trazer à tona alguns dos aspectos positivos das legendas interlinguais, nomeadamente os relacionados com o desenvolvimento da competência intercultural.

Nas últimas décadas, diversos estudos (VANDERPLANK, 1994; RUBIN, 1995; PAIVIO, 1986; LAMBERT \& HOLOBOW, 1984; DANAN, 2004; DÍAZ CINTAS, 2008) analisaram os efeitos positivos que o visionamento de materiais audiovisuais legendados na língua materna do aprendiz pode ter na aquisição. Entre esses efeitos, podemos destacar o fato de que eles favorecem a aquisição incidental, pois apresentam amostras autênticas do uso da língua em contextos autóctones, constituindo um insumo natural - pérola do ensino comunicativo - que a maioria dos espectadores processa sem precisar de nenhum esforço sistemático consciente. Além disso, a utilização deste recurso ajuda a desenvolver estratégias cognitivas úteis à comprovação de hipóteses, como adivinhação, inferência, clarificação e verificação de significados (RUBIN, 1995, apud DANAN, 2004).

Evidentemente, para a experiência ser bem-sucedida, os espectadores devem estar familiarizados com o visionado de programas ou filmes legendados, pois se requer 
uma série de estratégias específicas que o espectador desacostumado geralmente não utiliza. Sobre a ativação destas estratégias, citamos Gorovitz:

Ver um filme legendado é uma atividade que demanda esforço e uma atenção redobrada. $O$ espectador não lê em sequência: são idas $e$ voltas permanentes entre imagem, som e legenda. Este vai-e-vem solicita atividades simultâneas, nas quais diferentes sentidos são colocados em uma interação inusitada. Contudo, o espectador "regular" conquistou paulatinamente o hábito da "leitura cinematográfica", exercitando um leque de atividades mentais na repetição da experiência e integrando-as ao conjunto de suas habilidades (GOROVITZ, 2006, p. 64).

Portanto, um ponto crucial para as legendas interlinguais serem favoráveis à aquisição é o espectador estar familiarizado com elas ou, caso contrário, só obterá um alto grau de distração e poucos ganhos linguísticos. Em um primeiro momento, o professor deve ajudar os usuários inexperientes a se tornarem espectadores "regulares", mediante uma introdução sistemática que promova uma atenção reflexiva tanto aos elementos orais quanto ao tex to escrito.

De acordo com Paivio (1986) e Danan (1992) o insumo audiovisual com legendas interlinguais está constituído de três sistemas independentes que se interrelacionam mediante associações triplas - de imagem, som (em uma língua) e texto (em outra língua) -, o que, de acordo com eles, pode melhorar o processamento e a memorização graças aos efeitos aditivos da imagem e da tradução. Quando a tradução faz o vínculo entre os dois sistemas verbais, os espectadores contam com mais vias de recuperação da informação e se beneficiam tanto dos elementos visuais quanto dos dois códigos verbais.

Por sua vez, na sua análise da natureza do texto audiovisual, Delabastita (1989, p. 101) faz uma pertinente distinção entre os elementos verbais e não verbais, evitando, dessa forma, que os signos da comunicação não verbal fiquem relegados à invisibilidade. O autor propõe uma divisão do texto audiovisual em quatro componentes básicos ${ }^{1}$ : dois sistemas de signos - verbal e não verbal - e dois canais de comunicação -

\footnotetext{
${ }^{1}$ Segundo Delabastita (1989), estes quatro componentes básicos constituem quatro tipos de signos fílmicos: signos verbais transmitidos por um canal acústico (diálogos), signos não verbais transmitidos por um canal acústico (ruído de fundo, música), signos verbais transmitidos por um canal ótico (créditos, cartelas, documentos mostrados na tela) e signos não verbais transmitidos por um canal ótico.
} 
acústico e ótico -, todos eles presentes em maior ou menor medida no texto audiovisual. Este modelo de análise parece-nos mais adequado ao nosso interesse de estudo.

Em resposta aos críticos que alegam que os aprendizes, ao ler na língua materna, deixam de prestar atenção ao diálogo original, Danan (2004) recorre a diversos estudos cognitivos que mostraram que a leitura das legendas é um comportamento automático que acontece de forma sistemática, sem interferir no processamento da trilha sonora. De fato, d'Ydewalle e Gielen (1992, p. 425, apud DANAN, 2004) assinalam que o espectador divide sua atenção entre as legendas e o som em função de suas necessidades, sendo que normalmente dedica mais tempo a ler as legendas para garantir o processamento de informação complexa, até quando existem incongruências entre o verbal e o escrito. Um grupo de pesquisadores (DE BOT et al., 1986) corroborou, mediante experiências com um telejornal que continha desvios de nível fonológico, gramatical, lexical ou informacional, que o processamento dos dois canais ocorre de forma simultânea.

Gorovitz, em seu estudo sobre a interação entre os filmes legendados e o espectador, faz referência aos processos cognitivos desta experiência fílmica:

Lembrando que tanto questões de ordem técnica e prática quanto cultural justificam a "deficiência" da legendagem, o espectador é levado a interferir para compensar tal ausência, colocando a serviço da obra seu potencial subjetivo para prover as necessidades da interação. Por tanto, o receptor atua por correspondência, tirando de seu próprio léxico cultural os elementos que não estão explicitamente sugeridos pelo filme assim como aqueles que escapam à tradução. Essa reconstrução dá-se por meio das capacidades mentais do sujeito, que, ao solicitar sua memória, imaginação e criatividade, transforma suas fantasias e pulsões em figuras apreensíveis (GOROVITZ, 2006, p. 23).

Além dos ganhos cognitivos, os filmes legendados podem fornecer, pelo seu poder motivador, vantagens de tipo afetivo (BORGES GOMES, 2006, p. 33), assim como promover melhoras na habilidade de leitura dos aprendentes-espectadores:

A melhora na habilidade de leitura pelo uso de filmes legendados pode se dar por meio da associação visual entre palavras e imagens, pelo desafio para ler com rapidez e identificar as palavras-chave, pela oportunidade para observar qualquer discrepância entre legenda e áudio e pela oportunidade para estudar a correspondência entre língua falada e escrita. A apresentação multisensorial da imagem, 
linguagem oral e palavras escritas simultaneamente nos filmes legendados favorece a aquisição de novos conceitos, ao mesmo tempo que fornece contexto para o uso das palavras, reforçando assim, a aquisição de novo vocabulário. Esta associação de áudio, imagens e escrita também pode beneficiar a alunos que usam estratégias de aprendizado diferentes (SPANOS; SMITH, apud BORGES GOMES, 2006, p. 33).

Com relação ao desenvolvimento das habilidades de compreensão e expressão oral (incluindo os códigos da comunicação verbal e não verbal), e à aquisição de léxico e elementos culturais, Díaz-Cintas é categórico ao afirmar:

Sem dúvida nenhuma, ver e ouvir filmes legendados pode contribuir não só para o desenvolvimento de habilidades lingüísticas, mas também para o aprendizado de elementos culturais de um modo bastante lúdico. A imagem permite observar elementos reais de comunicação como a relação entre língua e gestos. A informação sonora contribui tanto para a entoação como para a pronúncia de palavras, as quais são importantes em idiomas como o inglês. As legendas, por sua vez, são redundantes na dimensão semântica e ajudam a ampliar o vocabulário do aprendiz (DÍAZ-CINTAS, apud BORGES GOMES, 2006, p. 13).

Reconhecidos os benefícios das legendas interlinguais, cabe-nos buscar propostas metodológicas adequadas para inserir este recurso na aula de língua estrangeira. Este artigo focará no seu potencial para desenvolver a competência intercultural do aprendente.

\section{Competência intercultural}

Como salienta Byram (1997), a comunicação bem-sucedida não é apenas aquela na qual ocorre um intercâmbio eficiente de informações, uma vez que, às vezes, uma mensagem claramente transmitida pode ser pragmaticamente inapropriada e provocar mal-entendidos e rupturas na comunicação. Portanto, a aquisição de uma nova língua requer a aquisição das práticas culturais e crenças a ela vinculadas, ainda que o aprendente não pretenda imitá-las. Este fator, essencial à manutenção das relações, não só depende dos conhecimentos do aprendente; também depende, em grande medida, das atitudes geradas. 
A competência intercultural será aqui tratada sob duas perspectivas: por um lado, como uma (sub)competência - tão importante quanto as outras - da competência comunicativa e, por outro, como uma competência que vai além da competência comunicativa, pois, sem uma competência comunicativa intercultural, a comunicação pode apresentar falhas, independentemente da competência comunicativa do indivíduo. Quando falamos do indivíduo, referimo-nos tanto ao aprendente de uma língua estrangeira quanto ao seu interlocutor, já que, em muitos casos, podem ocorrer rupturas na comunicação, não por causa de deficiências comunicativas do aprendiz, mas pela falta de uma competência intercultural do falante nativo com o qual está tentando interagir. $\mathrm{O}$ mesmo pode acontecer entre falantes da mesma cultura.

A respeito disso, Byram e Kramsch criticam o modelo de ensino de línguas que toma o falante nativo como modelo a seguir, porque tal procedimento pode provocar sentimentos conflitantes no aprendiz, que, ao pressupor que deve imitar com perfeição tudo o que o outro fala e faz, sofre frustrações e teme pela integridade de sua identidade - o que Byram (1997, p. 11) chama de "esquizofrenia linguística". O autor nos lembra que, quando um aprendente de uma língua estrangeira interage com um falante nativo dessa cultura, não deve vê-lo como alguém superior, mas como um igual que fala uma língua diferente e vive inserido em uma outra cultura - nem inferior nem superior à dele -, com a qual vai comunicar-se sem precisar renunciar à sua identidade cultural.

Apesar das limitações de um modelo fracionado de competências, que parece uma tentativa inútil de dividir um fenômeno dinâmico em categorias estanques, nosso objetivo é fazer uma delimitação aproximada da competência intercultural para fins descritivos. A partir desta perspectiva, pode-se dizer que a competência intercultural se constitui de três elementos básicos (BYRAM, 1997, pp. 35-38):

- $\quad$ um saber ser, que tem a ver com as atitudes do falante e consiste em sua capacidade de estabelecer uma relação entre sua cultura e outras culturas, relativizando a experiência própria e valorizando as alheias;

- $\quad$ os saberes que estruturam o conhecimento explícito e implícito sobre si mesmo, sobre o outro e sobre os processos de interação, adquiridos durante a aprendizagem linguística-cultural;

- uma série de capacidades, desmembradas nos seguintes saberes: 
- $\quad$ saber compreender, que consiste na capacidade de interpretar e relacionar documentos ${ }^{2}$ ou eventos;

- saber engajar-se, o que depende da educação política e da consciência cultural crítica do falante para avaliar de forma crítica os pontos de vista, práticas e produtos da sua cultura e das outras; e - saber fazer ou saber aprender, que se refere à capacidade de descobrir fenômenos significativos das culturas e de interagir com o outro.

Em suma, um falante com competência intercultural tem a capacidade de desenvolver-se adequada e satisfatoriamente nas situações de comunicação intercultural que caracterizam o mundo atual, onde são crescentes os movimentos transnacionais e as sociedades multiculturais. Como preconiza o saber engajar-se, o aprendente não precisa imitar todos os comportamentos observados na outra cultura, mas saber analisá-los e entender os diferentes pontos de vista.

Conforme este princípio, o falante intercultural, ao interagir com o outro, negocia significados dando lugar ao que Kramsch (1993) denomina "terceiro espaço", uma terceira cultura que não corresponde à cultura do aprendente nem à outra cultura, mas que surge, num processo dialógico, nos interstícios delas. A autora ressalta que o professor de línguas não deve fazer uma ponte entre as diferenças para anulá-las, criando uma falsa ideia de universalidade, e sim propiciar um contexto dialógico no qual se explorem as diferenças e se reconfigurem as bases ideológicas da divisão e da diferença.

No entanto, essa terceira cultura não emerge de forma automática na sala de aula. Kramsch (apud LIDDICOAT; CROZET; BIANCO, 1999, p. 181) propõe estratégias para que o docente propicie oportunidades para os alunos atuarem como mediadores entre sua cultura e as outras. Já que os métodos, o currículo e os materiais didáticos são elementos que influenciam na criação deste terceiro espaço, uma abordagem intercultural deve ser adotada de forma consistente para contribuir com o sucesso da formação de mediadores interculturais.

\footnotetext{
${ }^{2}$ Acreditamos que o autor utiliza o termo 'document' como sinônimo de texto (p. ex. material didático ou texto lido fora da aula), mas também com a acepção de fonte fidedigna susceptível de ser usada para provar algo. Para ele, uma matéria de jornal e um texto literário são documentos. O evento seria o fato sobre o qual falam os documentos.

${ }^{3}$ Conceito cunhado por Bhabha ([1992] 1998) para referir-se a um entre-lugar imaginário onde o sujeito reformula sua identidade a partir de um encontro dialógico com a alteridade.
} 


\section{O saber compreender e a tradução}

A tradução é considerada por alguns linguistas aplicados como uma 'quinta habilidade' linguística, junto com as habilidades de compreensão oral, compreensão leitora, expressão oral e expressão escrita. Newmark (1987/1992), que defende o uso da tradução em todos os estágios do processo de ensino e aprendizagem de uma língua estrangeira ou segunda, apresentou um modelo com diferentes tipos de tradução para diferentes níveis de aprendizagem.

Em seus estudos, Byram (1997, p. 37) cita a tradução como um ato comunicativo no qual todo falante intercultural pode ver-se envolvido. Como explica o autor, este ato de mediação requer a capacidade de interpretar - com ajuda de informação específica e quadros gerais de conhecimento - as alusões e conotações do documento. A capacidade de interpretar, traduzir e relacionar documentos de outros países depende, portanto, do conhecimento explícito e implícito do indivíduo sobre sua cultura e aquela à qual quer aceder, assim como de sua capacidade para analisar as disfunções, contradições e questões impossíveis de resolver.

Vale ressaltar a influência que exercem os tópicos e estereótipos na interpretação dos documentos, e aí reside a importância de estabelecer relações com outros documentos, com outros olhares, e questionar sempre o (aparentemente) óbvio. Esta tarefa cabe tanto aos aprendentes quanto ao professor, que também precisa desenvolver sua competência intercultural. Em muitas ocasiões, o próprio professor é surpreendido com as descobertas dos alunos, que podem apresentar uma visão de sua cultura até então desconhecida para ele. Da mesma maneira, os aprendentes, ao olharem para a alteridade, podem descobrir características de sua própria cultura e reformular sua identidade cultural.

Por esse motivo, os conteúdos culturais não podem ser simplesmente transmitidos como se fossem fórmulas matemáticas. Byram nos adverte sobre esta questão:

Devemos estar conscientes dos perigos que acarreta apresentar 'uma cultura' como algo imutável ou como se fosse unicamente um conjunto de crenças, significados e comportamentos de um determinado país. Quando os indivíduos interagem, trazem para a situação suas identidades e culturas próprias, e se não são membros 
de um grupo dominante, subscritos à cultura dominante, o conhecimento do seu interlocutor sobre essa cultura será disfuncional. (BYRAM, 1997, p. 39)

O professor que busque propiciar o diálogo e facilitar acessos a terceiros espaços, deve escolher com extremo cuidado os materiais didáticos que vai utilizar para abordar aspectos culturais específicos. Textos com afirmações e generalizações não são apropriados, pois impõem a priori um ponto de vista - geralmente, o da cultura dominante - que leva à manutenção dos estereótipos. Para garantir a função emancipadora da educação, é fundamental manter uma relação de respeito e igualdade entre os agentes envolvidos, os quais devem procurar desenvolver suas capacidades de descoberta e interpretação da alteridade de uma maneira reflexiva. Neste sentido, a tradução apresenta-se como o espaço privilegiado para que se desenvolvam estas capacidades de maneira autônoma, crítica e reflexiva.

O aprendente de uma língua estrangeira que faz o papel de mediador coloca-se em um lugar de leitor privilegiado, uma vez que, ao tentar encontrar equivalências entre as duas linguaculturas, ${ }^{5}$ atinge uma compreensão maior sobre sua linguacultura e sobre a linguacultura a que ele quer aceder. É especialmente a partir das diferenças que surge a reflexão identitária e a necessidade de compreender o outro.

Na mesma linha, Gorovitz (2006, p. 56) explica: "Em sua definição mais ampla, a tradução é a atividade de compartilhar por excelência. A leitura que se faz do outro é sempre uma leitura de si, renovada pela diferença do objeto dessa leitura". Isso significa que o indivíduo é inevitavelmente tradutor de sua própria relação com o mundo e, através deste ato, transforma e constrói aquilo que só pode existir por sua apropriação subjetiva.

$\mathrm{Na}$ presente proposta de trabalhar com materiais audiovisuais legendados na língua dos aprendizes, eles não atuam diretamente como tradutores, mas sim como usufrutuários da tradução. Por isso, concordamos com Gorovitz (2006, p. 56) quando observa que:

\footnotetext{
${ }^{4}$ We have to be aware of the dangers of presenting 'a culture' as if it were unchanging over time or as if there were only one set of beliefs, meanings and behaviours in any given country. When individuals interact, they bring to the situation their own identities and cultures and if they are not members of a dominant group, subscribing to the dominant culture, their interlocutor's knowledge of that culture will be dysfunctional.

${ }^{5} \mathrm{O}$ conceito 'linguacultura', cunhado por Attinasi e Friedrich em 1988, é aqui usado para enfatizar a indivisibilidade entre língua e cultura.
} 
"Traduzir o filme significa traduzir o outro; ler o filme traduzido também implica traduzir traduções". De acordo com esta afirmação, o uso de filmes com legendas interlinguais pode ser um excelente estímulo para desenvolver a capacidade de mediação intercultural do aprendente.

\section{A comunicação não verbal}

Alguns autores apontam para a necessidade de incluir a comunicação não verbal no ensino de língua estrangeira, pois muitos mal-entendidos interculturais devem-se ao fato de que os códigos da comunicação não verbal variam de cultura para cultura. Poyatos (1992, apud BYRAM, 1997, p. 13) critica a maneira como vem sendo ignorado este elemento da comunicação na sala de aula e afirma que todo professor deveria levar em conta a tripla realidade do discurso (composto por linguagem, paralinguagem e cinética).

De acordo com Poyatos (1994), uma cultura começa a se desenvolver no momento em que há um intercâmbio interativo entre, pelo menos, dois organismos socializantes entre os quais se produz uma emissão-recepção de signos. Esta interação apresenta, como indica o autor, os seguintes contextos comunicativos: (1) comunicação vocal e verbal (linguagem); (2) comunicação vocal e não verbal (paralinguagem); e (3) comunicação não vocal e não verbal (kinésica, proxêmica e os demais sistemas corporais). Por essa razão, o aprendente de uma língua estrangeira deve preparar-se para manter interações complexas onde todos estes códigos confluem.

Byram (1997, p. 47) também destaca a importância de conhecer todos os elementos da interação, mas alerta para o fato de que, ao ser muitos aspectos da comunicação não verbal adquiridos inconscientemente dentro de um meio cultural específico, é provável que o aprendiz não chegue a dominá-los ou que não queira renunciar aos que possui, tão enraizados que até fazem parte de sua personalidade. Mais uma vez, o autor chama a atenção para a necessidade de se preparar o aprendiz para compreender e interpretar os códigos da outra cultura sem pretender que os incorpore na sua linguagem. 
Dada a importância de se trabalhar a comunicação não verbal na aula de língua estrangeira, é inquestionável a potencialidade do uso dos materiais audiovisuais para este fim. A mensagem fílmica está permeada de uma série de elementos prosódicos, paralinguísticos, proxêmicos e kinésicos que transmitem uma grande quantidade de informação: pausas, repetições, entonações, tons, interrupções, variações na velocidade de articulação, expressões faciais, gestos, dentre outros. As variações dialectais também carregam um valor social e cultural que muitas vezes é incompreendido ou passa despercebido pelo espectador estrangeiro.

Ao assistir a filmes legendados, o espectador baseia-se na imagem e no som para captar estes signos, pois as legendas não são suficientes para traduzir todos eles. Uma das dificuldades do tradutor de legendas é conjugar sua tradução com o que aparece na tela. Ao traduzir os diálogos, quem traduz não deve negligenciar o poder semiótico da imagem, uma vez que pode deparar-se com gestos ou comportamentos não verbais diferentes dos códigos conhecidos pelo espectador. Por exemplo, um assentimento de cabeça pode ter significados opostos dependendo da cultura onde este gesto seja realizado: enquanto nas culturas ocidentais tem um uso generalizado com sentido de afirmar, no norte da Índia e na Bulgária se utiliza como negação. Diferenças deste tipo podem ser evidenciadas ao analisar um filme legendado, na aula de língua estrangeira, sobretudo quando as legendas parecem contradizer a imagem. Cabe, portanto, ao professor escolher com cuidado as cenas que serão trabalhadas com os alunos para garantir um maior aproveitamento deste recurso.

\section{Bases metodológicas: por uma competência comunicativa intercultural}

Apesar de contarmos com uma (modesta) produção científica, na qual se abordam os efeitos positivos das legendas interlinguais na aquisição de línguas, ainda se requerem esforços para elaborar propostas metodológicas que incluam este recurso. Zabalbeascoa (1990, p. 82) apresenta algumas ideias para trabalhar as quatro habilidades linguísticas mediante tarefas baseadas na tradução pedagógica; ele propõe, por exemplo, que se realizem atividades envolvendo filmes em versão original com legendas na L1 como exercício para desenvolver a compreensão oral, e atividades com 
filmes na L1 legendados na outra língua para desenvolver a compreensão leitora do aprendente.

No entanto, o leque de possibilidades abre-se muito mais se partimos de uma proposta com foco nas capacidades descritas por Byram e os elementos da comunicação não verbal apresentados por Poyatos, ampliando, assim, o escopo da competência comunicativa para a competência intercultural. Desta forma, é possível elaborar uma proposta metodológica a partir do modelo sugerido por Byram (1997, pp. 57-64), no qual ele estabelece uma série de objetivos:

Atitudes: curiosidade, abertura e disposição para dar credibilidade a outras culturas e desacreditar a própria.

Objetivos:

- vontade de procurar ou aproveitar oportunidades para estabelecer uma relação equitativa com o outro; não deve confundirse com a vontade de procurar o exótico e tirar proveito do outro;

- $\quad$ interesse por conhecer outros pontos de vista na interpretação de fenômenos e práticas culturais da própria cultura e das outras culturas;

- $\quad$ vontade de questionar valores e pressupostos sobre práticas culturais e produtos da própria cultura;

- disposição para envolver-se nos ritos e convenções da comunicação verbal e não verbal e na interação.

Conhecimento: de grupos sociais e suas práticas e produtos no próprio país e no país do interlocutor, bem como dos processos gerais de interação individual e social.

Objetivos (conhecimento sobre/de):

- relações históricas e contemporâneas entre o próprio país e o país do interlocutor;

- os meios para fazer contato com interlocutores de outro país (distante ou próximo), para viajar para ou desde lá, e sobre as instituições que facilitam o contato ou ajudam a resolver problemas;

- $\quad$ as causas e os processos de mal-entendidos entre interlocutores de origens culturais diversas;

- $\quad$ a memória nacional do próprio país e a maneira como esses eventos são vistos pelo país do interlocutor e se relacionam com ele;

- $\quad$ a memória nacional do país do interlocutor e a maneira como ele é visto pelo próprio país;

- $\quad$ as definições nacionais de espaço geográfico no próprio país e a percepção que delas se tem por parte dos outros países;

- as definições nacionais de espaço geográfico no país do interlocutor e a perspectiva que delas se tem no próprio país;

- $\quad$ os processos e instituições de socialização no próprio país e no país do interlocutor;

- $\quad$ as distinções sociais e seus principais indicadores no próprio país e no país do interlocutor; 
- $\quad$ as instituições - e a percepção que delas se tem-que afetam a vida diária do próprio país e do país do interlocutor e que guiam e influenciam as relações entre eles;

- $\quad$ os processos de interação social no país do interlocutor.

Capacidades de interpretação e relação: capacidade para interpretar um documento ou um evento de outra cultura, para explicá-lo e relacioná-lo a documentos da própria cultura.

Objetivos (capacidade para):

- identificar perspectivas etnocêntricas em um documento ou evento e explicar suas origens;

- identificar em uma interação áreas de mal-entendidos e disfunções e explicá-los em função dos sistemas culturais envolvidos;

- $\quad$ mediar entre interpretações conflitantes de um fenômeno.

Capacidades de descoberta e interação: capacidade para adquirir novos conhecimentos sobre as práticas culturais e para utilizar o conhecimento, as atitudes e as capacidades dentro das limitações da comunicação e da interação em tempo real.

Objetivos (capacidade para):

- $\quad$ obter do interlocutor os conceitos e valores de documentos ou eventos, e desenvolver um sistema explicativo susceptivel de ser aplicado a outros fenômenos;

- identificar referências significativas em e entre culturas, e descobrir sua importância e conotações;

- $\quad$ identificar processos similares e diferentes de interação, verbal e não verbal, e negociar um uso apropriado deles em situações específicas;

- usar, em tempo real, uma combinação adequada de conhecimentos, capacidades e atitudes para interagir com interlocutores de um outro país e cultura, levando em consideração o grau de familiaridade que se tem com o país/cultura e o grau de distanciamento cultural que existe entre as duas culturas.

- identificar relações passadas e contemporâneas entre seu país/cultura e o país/cultura do outro;

- $\quad$ identificar e fazer uso de instituições públicas e privadas que facilitam o contato com outros países e culturas;

- $\quad$ usar, em tempo real, os conhecimentos, capacidades e atitudes para mediar entre interlocutores da própria cultura e da cultura estrangeira.

Consciência cultural críticaleducação política: capacidade para analisar de forma crítica, com base em critérios explícitos, os pontos de vista, práticas e produtos do próprio país/cultura e de outros países/culturas.

Objetivos (capacidade para):

- identificar e interpretar valores explícitos e implícitos de documentos e eventos do próprio país e de outros países.

- $\quad$ fazer uma avaliação dos documentos e eventos a partir de um ponto de vista e de certos critérios explícitos; 
- $\quad$ interagir e mediar em encontros interculturais de acordo com critérios explícitos negociando, quando necessário, sua aceitação fazendo uso dos conhecimentos, capacidades e atitudes.

Os objetivos aqui expostos, baseados na competência intercultural, podem ser integrados aos objetivos habituais do ensino comunicativo, ou seja, àqueles voltados para o desenvolvimento das competências linguística, sociolinguística, discursiva e estratégica, construindo, assim, um currículo propício para o desenvolvimento da competência comunicativa intercultural.

Como em toda atividade docente, o contexto social e político em que trabalha o professor e as características individuais e coletivas dos alunos são elementos que influenciam na Operação Global de Ensino de Língua(s) ${ }^{6}$. Portanto, não é o objetivo deste artigo apresentar uma proposta metodológica concreta para trabalhar com legendas interlinguais, e sim algumas orientações que possam servir como base para a elaboração de programas, materiais didáticos e planos de aula - incluindo o uso de materiais legendados - que visem a uma formação dos discentes como falantes e mediadores interculturais.

O modelo por objetivos, conforme apresentado por Byram, propõe-se a considerar a comunicação e a interação intercultural na sua complexidade. Para o autor (1997, p. 29), um modelo baseado em categorias mensuráveis da competência comunicativa intercultural é vantajoso para efeitos de certificação, mas toda tentativa de fazer uma avaliação objetiva das capacidades do aprendente pode levar a uma excessiva simplificação e a uma inadequada representação das mesmas. Por esse motivo, só é recomendável usar um modelo por níveis de competência em casos específicos de avaliação.

Como ponto de partida, damos alguns exemplos de atividades que, bem elaboradas e inseridas em unidades didáticas que integrem as cinco habilidades linguísticas, podem ser úteis aos nossos objetivos, a saber:

- Analisar a relação entre os quatro sistemas semióticos do filme legendado e extrair diferenças linguísticas, pragmáticas ou contextuais existentes

\footnotetext{
${ }^{6}$ Conceito criado por Almeida Filho (1993) para referir-se de uma maneira holística ao processo de ensino e aprendizagem de línguas e às diversas forças que o compõem.
} 
entre a cultura do filme e a dos aprendentes (isto pode gerar atividades diferentes, como as focadas nos códigos da comunicação não verbal).

- Analisar (interpretar de forma crítica) as estratégias utilizadas pelo tradutor para legendar cenas de humor, e refletir sobre a adequação da tradução e sobre a existência de outras possibilidades de aproximação à cultura dos aprendentes.

- Analisar diferenças linguísticas e de registro entre a tradução e os diálogos em cenas onde predomina o uso coloquial da língua (palavrões, gírias, socioletos, etc.), e comparar esses usos sociais com os da própria cultura.

- Analisar inadequações gramaticais, semânticas e pragmáticas de tradução em cenas legendadas, e pensar nas causas e em possíveis soluções.

- $\quad$ Analisar estratégias de tradução utilizadas pelo legendador para amenizar ou transgredir uma visão imperialista do filme ou programa.

- Analisar, a partir das legendas, a 'intraduzibilidade' de algumas referências culturais características da cultura do filme (alimentos, folclore, fauna e flora, por exemplo) e tentar entender, com base no contexto fílmico, o valor que estas referências possuem no seu contexto cultural e o valor que teriam na cultura dos aprendentes.

- Observar os estereótipos mostrados no filme - sobre qualquer cultura - e procurar maneiras de avaliar se esses estereótipos refletem realmente os comportamentos dessa cultura.

- Analisar algumas estratégias de tradução que são características da legendagem e que podem ser empregadas na comunicação ou mediação intercultural, como as estratégias de omissão, compensação, adaptação, modulação, compressão, criação discursiva, generalização, redução, tradução literal etc.

Vale ressaltar que as atividades aqui sugeridas não possuem o objetivo de avaliar a habilidade tradutória dos aprendentes - dadas suas complexidades -, mas foram pensadas para promover a interpretação, descoberta e reflexão crítica das culturas a partir de uma abordagem intercultural. Neste contexto, estamos falando de uma modalidade de tradução pedagógica, utilizada como um meio e como um fim, pois 
consideramos que ela ajuda a desenvolver uma das cinco habilidades linguísticas envolvidas na comunicação: a da tradução.

\section{Considerações finais}

Diante do que acabamos de apresentar, acreditamos que o uso pedagógico de materiais audiovisuais com legendas interlinguais pode contribuir para o desenvolvimento da competência comunicativa e da competência intercultural. Nossa expectativa é que as reflexões aqui apresentadas possam servir para que profissionais e pesquisadores da Linguística Aplicada avaliem a utilização deste recurso didático, ainda pouco aproveitado, como um material (ou documento) de inquestionável potencial para o ensino-aprendizagem de língua estrangeira ou segunda língua.

Acreditamos que futuras pesquisas poderão contribuir para a valorização e o reconhecimento desta área fértil, ajudando a consolidar nossa proposta neste artigo: a desmistificação da tradução, em todas suas modalidades, como erva daninha do nosso frutífero jardim - a aula de língua estrangeira.

\section{Referências}

ALMEIDA FILHO, José Carlos P. Dimensões comunicativas no ensino de línguas. Campinas: Pontes, 1993.

ATTINASI, John; FRIEDRICH, Paul. Dialogic breakthrough: catalysis and synthesis in life-changing dialogue. In: MANNHEIM, Bruce; TEDLOCK, Dennis (Eds.). Toward a dialogic Anthropology. [s.1.], 1988.

BHABHA, Homi K. O local da cultura. Tradução de Myriam Ávila, Eliana Lourenço de Lima Reis e Gláucia Renate Gonçalves. Belo Horizonte: UFMG, 1998.

BORGES GOMES, Francisco W. O uso de filmes legendados como ferramenta para o desenvolvimento da proficiência oral de aprendizes de língua inglesa. Dissertação (Mestrado em Linguística Aplicada) - Universidade Estadual do Ceará, 2006.

BYRAM, Michael. Teaching and assessing intercultural communicative competence. Clevedon: Multilingual Matters, 1997. 
CARR, Jo. From 'sympathetic' to 'dialogic' imagination: cultural study in the foreign language classroom. In: BIANCO, Joseph L., LIDDICOAT, Anthony J.; CROZET, Chantal (Eds.). Striving for the third place: intercultural competence through language education. Melbourne: Language Australia, 1999. p. 103-112.

COSTA, Walter C. Tradução e ensino de línguas. In: BOHN, Hilário; VANDRESEN, Paulino (Orgs.). Tópicos de linguística aplicada. Florianópolis: UFSC, 1988.

DANAN, Martine. Reversed subtitling and dual coding theory: New directions for foreign language instruction. Language Learning, vol. 42, n. 4, p. 497-527, 1992.

Captioning and subtitling: undervalued language learning strategies. Meta: Journal des Traducteurs / Meta: Translators'Journal, n. 1, p. 67-77, 2004.

DE BOT, Kees; JAGT, John; JANSSEN, Henk; KESSELS, Erik; SCHILS, Erik. Foreign television and language maintenance. Second Language Research, vol. 2, n. 1, p. 72-82, 1986.

DELABASTITA, Dirk. Translation and mass-communication: film and T.V Translation as evidence of cultural dynamics, Babel, vol. 3, n. 4, p. 193-218, 1989.

DÍAZ CINTAS, Jorge. The didactics of audiovisual translation. Amsterdam/Philadelphia: John Benjamins, 2008.

FERREIRA, Sonia Maria G. Following the paths of translation in language teaching: from disregard in the past to revival towards the 21st century. Cadernos de Tradução, vol. 4, p. 355-371, 1999.

GOROVITZ, Sabine. Os labirintos da tradução: a legendagem cinematográfica e a construção do imaginário. Brasília: Universidade de Brasília, 2006.

HURTADO ALBIR, Amparo. Enseñar a traducir. Madrid: Edelsa, 1999.

KRAMSCH, Claire. Context and culture in language teaching. Oxford: Oxford University Press, 1993.

LAMBERT, Wallace E.; HOLOBOW, Naomi E. Combinations of printed script and spoken dialogues that show promise for beginning students of a foreign language. Canadian Journal of Behavioural Science, vol. 16, p. 1-11, 1984.

LAVAULT, Elisabeth. Fonction de la traduction en didactique des langues. Paris: Payot, 1998.

LIDDICOAT, Anthony J.; CROZET, Chantal; BIANCO, Joseph L. Striving for the third place: consequences and implications. In: BIANCO, Joseph L., LIDDICOAT, Anthony J.; CROZET, Chantal (Eds.). Striving for the third place: intercultural competence through language education. Melbourne: Language Australia, 1999. p. 103112. 
NEWMARK, Peter. Manual de Traducción. Tradução de Virgilio Moya. Madrid: Cátedra, 1995.

PAIVIO, Allan. Mental representation: a dual-coding approach. New York: Oxford University Press, 1986.

POPOVIC, Radmila. The place of translation in language teaching. English Teaching Forum, vol. 37, n. 2, 1999.

POYATOS, Fernando. La comunicación no verbal: cultura, lenguaje y conversación, v. 1. Madrid: Istmo, 1994.

RIDD, Mark. D. Um casamento estranhamente ideal? A compatibilidade de gênios entre o comunicativismo e a tradução. Horizontes de Linguística Aplicada, vol. 2, n. 1, p. 93$104,2003$.

RUBIN, Joan. Learner processes and learner strategies. In: GALLOWAY, V.; HERRON, C. (Eds.). Research within research II. Valdosta, GA: SCOLT, 1995.

VANDERPLANK, Robert. Resolving inherent conflicts: autonomous language learning from popular broadcast television. In: JUNG, Heidrun; VANDERPLANK, Robert (Eds.). Barriers and Bridges: Media Technology in Language Learning: Proceedings of the 1993 CETaLL Symposium on the Occasion of the 10th AILA World Congress in Amsterdam, Peter Lang, Frankfurst, pp. 119-134, 1994.

VANDERPLANK, Robert. Resolving inherent conflicts: autonomous language learning from popular broadcast television. In:

ZABALBEASCOA TERRÁN, Patrick. Aplicaciones de la traducción a la enseñanza de lenguas extranjeras. Sintagma, vol. 2, p. 75-86, 1990. 